\title{
Diurnal Profiles of Physical Activity and Postures Derived From Wrist-Worn Accelerometry in UK Adults
}

\author{
Ignacio Perez-Pozuelo, Thomas White, Kate Westgate, Katrien Wijndaele, \\ Nicholas J. Wareham, and Soren Brage \\ University of Cambridge
}

\begin{abstract}
Background: Wrist-worn accelerometry is the commonest objective method for measuring physical activity in large-scale epidemiological studies. Research-grade devices capture raw triaxial acceleration which, in addition to quantifying movement, facilitates assessment of orientation relative to gravity. No population-based study has yet described the interrelationship and variation of these features by time and personal characteristics. Methods: 2,043 United Kingdom adults (35-65 years) wore an accelerometer on the non-dominant wrist and a chest-mounted combined heart-rate-and-movement sensor for 7 days free-living. From raw $(60 \mathrm{~Hz})$ wrist acceleration, we derived movement (non-gravity acceleration) and pitch and roll (forearm) angles relative to gravity. We inferred physical activity energy expenditure (PAEE) from combined sensing and sedentary time from approximate horizontal arm angle coupled with low movement. Results: Movement differences by time-of-day and day-ofweek were associated with forearm angles; more movement in downward forearm positions. Mean $(S D)$ movement was similar between sexes $\sim 31$ (42) $\mathrm{m} g$, despite higher PAEE in men. Women spent longer with the forearm pitched $>0^{\circ}$, above horizontal $(53 \%$ vs $36 \%)$, and less time at $<0^{\circ}(37 \%$ vs $53 \%)$. Diurnal pitch was $2.5-5^{\circ}$ above and $0-7.5^{\circ}$ below horizontal during night and daytime, respectively; corresponding roll angles were $\sim 0^{\circ}$ (hand flat) and $\sim 20^{\circ}$ (thumb-up). Differences were more pronounced in younger participants. All diurnal profiles indicated later wake-times on weekends. Daytime pitch was closer to horizontal on weekdays; roll was similar. Sedentary time was higher (17 vs 15 hours/day) in obese vs normal-weight individuals. Conclusions: More movement occurred in forearm positions below horizontal, commensurate with activities including walking. Findings suggest time-specific population differences in behaviors by age, sex, and BMI.
\end{abstract}

Keywords: BMI, movement, PAEE, pitch angle, roll angle, sedentary behavior, signal processing

Wrist-worn accelerometry has become a feasible option for the objective measurement of physical activity in large-scale epidemiological studies, such as Pelotas birth cohorts, the UK Biobank, and Whitehall II (da Silva et al., 2014; Doherty et al., 2017; Menai et al., 2017). Additionally, public adoption of consumer-grade wearable devices that include accelerometry has been increasing steadily in recent years (Hui-Wen Chuah et al., 2016; Lamkin, 2016; Li, Wu, Gao, \& Shi, 2016), with potential utility for public health research (White, Westgate, Wareham, \& Brage, 2016).

Accelerometers record a continuous time-series of data and recent advances in the technology and battery life allow for ubiquitous capture of raw accelerometer signals which have the potential to provide insights to interventional and epidemiological studies. Several features can be easily extracted from the acceleration signal, including the magnitude of movement and the orientation of the accelerometer with respect to gravity.

(C) 2020 The Authors. Published by Human Kinetics, Inc. This is an Open Access article distributed under the terms of the Creative Commons Attribution 4.0 International License, CC BY 4.0, which permits unrestricted noncommercial and commercial use, distribution, and reproduction in any medium, provided the original work is properly cited, the new use includes a link to the license, and any changes are indicated. See http://creativecommons.org/licenses/by/4.0. This license does not cover any third-party material that may appear with permission in the article.

The authors are with the MRC Epidemiology Unit, University of Cambridge, Cambridge, United Kingdom. Brage (soren.brage@mrc-epid.cam.ac.uk) is corresponding author.
Previous research using wrist accelerometry has described variation in population physical activity expressed predominantly as the activity-related acceleration magnitude. For example, da Silva et al noted age and sex differences in three Brazilian birth cohorts from Pelotas assessed at the ages 6, 18, and 30 years of age (da Silva et al., 2014), and Doherty et al described the unique diurnal patterns of physical activity by age group, documenting that the lower activity levels generally observed in older adults are particularly pronounced in the later hours in the day (Doherty et al., 2017). Magnitude-based measures of activity have also been related to health outcomes, such as body composition and fitness (Cho \& Kim, 2017; White et al., 2016).

Less attention has been given to the description of orientationrelated measures of human behavior using wrist-worn accelerometry, although they have been used in thigh-worn activity monitoring (Steeves et al., 2015). Pitch and roll angles are examples of well-defined, biomechanically relevant, and easy-tointerpret signal features that describe device orientation. In Figure 1, we illustrate pitch and roll for an individual with an accelerometer placed on the left wrist, and axes aligned as shown. Body posture is by definition a description of angles of all segments of the body and is determined by their relationship with gravity, which can in theory all be measured but in practice usually are not in studies of free-living behavior. However, as body segments are connected, and therefore range of motion is restricted, measurements and the variables that can be mathematically derived from them tend to be highly correlated (White et al., 2019). This allows inferences from the measurement of one body site to be made 


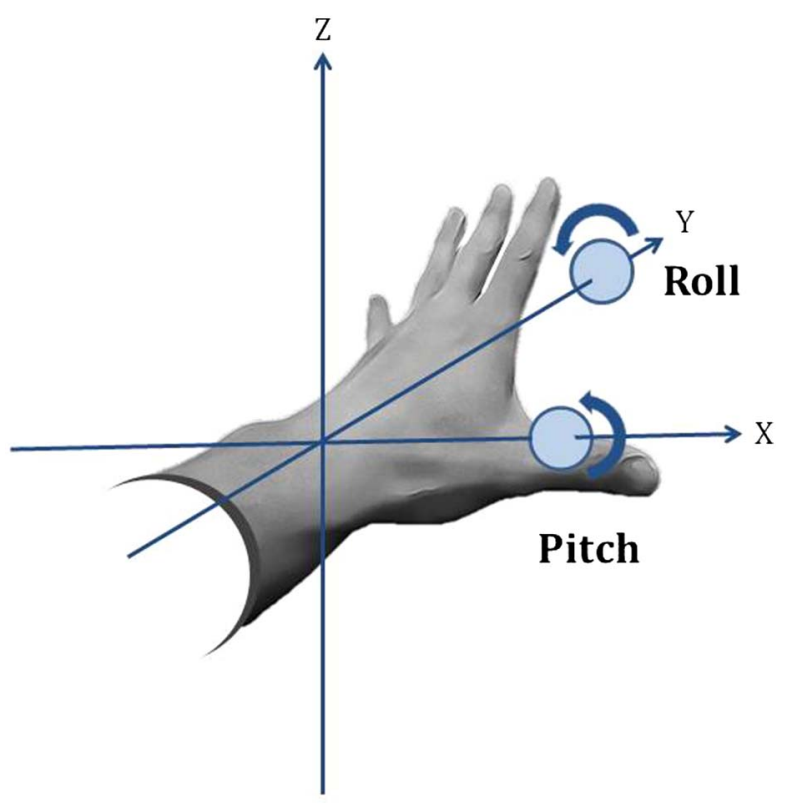

Figure 1 - Schematic of forearm Pitch and Roll on participant with accelerometer on the left wrist, including axes alignment. Roll is defined by rotation around the $y$-axis, while Pitch is defined by rotation around the $x$-axis. (Note that axis labeling depends on study protocol and device specifications).

on whole-body posture. For example, previous work has shown strong correlations between time spent sedentary inferred from wrist accelerometry (by combining information on acceleration magnitude and pitch angle) and thigh accelerometry $(r=\sim 0.93)$ (Rowlands et al., 2016).

Sedentary behavior can be defined as any waking behavior that is characterized by an energy expenditure $\leq 1.5$ METs while the subject is engaging in either sitting, lying, or reclining postures (Tremblay et al., 2017). People spend the majority of their time in sedentary behaviors, and the proportion of time spent sedentary increases as people age (Matthews et al., 2008). High volumes of sedentary behavior have been associated with increased mortality and risk of developing chronic conditions (Dunstan, Howard, Healy, \& Owen, 2012; Ekelund et al., 2016; Matthews et al., 2008; Patterson et al., 2018; Tremblay et al., 2017). This only seems to be eliminated by very high levels of moderate-intensity physical activity (60-75 min per day; i.e., equivalent to double the amount currently recommended for adults [Ekelund et al., 2016]). However, most of this evidence base is based on self-reported sedentary and activity estimates which come with important methodological limitations and bias (van der Ploeg \& Hillsdon, 2017).

Consequently, objectively assessing sedentary behaviors, as well as characterizing different activities performed during daily living, may be critical to inform public health recommendations. Traditionally, sedentary and active behaviors were characterized using such intensity derived measures from the accelerometer signal. Supplementary Figure 1 (available online) provides a visual representation of triaxial wrist acceleration (top panels) during four common activities of lying, walking, sitting, and cycling, alongside derived pitch and roll angles (bottom panels), demonstrating clear differences between activity types. When assessing activity patterns, diurnal profiles of pitch and roll combined with movement intensity metrics may allow us to further understand how different postures relate to different activities and activity intensities.
In this study, we aimed to describe the distribution of forearm postures, acceleration, derived sedentary time, and physical activity energy expenditure (PAEE) in a large cohort of United Kingdom (UK) adults ( $n=2,043$ participants). These analyses allow us to further understand the distribution of sedentary and active behaviors in the population and how this distribution may differ based on time of the day, sex, age, body mass index (BMI), and overall activity levels. Ultimately, the methodology developed for the work presented aims to help inform how changes in sedentary and active behaviors may impact energy expenditure.

\section{Methods}

\section{Study Population}

The Fenland Study is an ongoing prospective cohort study of 12,435 men and women aged 35-65 years, designed to identify the behavioral, environmental, and genetic causes of obesity and type-2 diabetes. As previously described in detail, participants attended one of three clinical research facilities in the region surrounding Cambridge, UK, and completed a series of physical assessments and questionnaires (O'Connor, Brage, Griffin, Wareham, \& Forouhi, 2015). Exclusion criteria for participation in the study were: clinically diagnosed diabetes mellitus, inability to walk unaided, terminal illness, clinically diagnosed psychotic disorder, pregnancy, or lactation. Following the baseline clinic visit, all participants were asked to wear a combined heart rate and movement sensor (Actiheart, CamNtech, Cambridgeshire, UK) for 6 consecutive days and nights, and a subsample of 2,100 participants were asked to simultaneously wear a wrist accelerometer (GeneActiv, ActivInsights, Cambridgeshire, UK) on the non-dominant wrist. This subsample constitutes the sampling frame for the current analyses. Participants were excluded from this analysis if they had insufficient individual calibration (treadmill testbased) data, or had less than 72 hours of concurrent wear data (equivalent of three full days of recording). Given only very few participants were very severely underweight $(B M I \leq 15)$ in this subset of the Fenland study, they were also excluded, resulting in a total of 2,043 subjects.

All participants provided written informed consent and the study was approved by the University of Cambridge research ethics committee and performed in accordance with the Declaration of Helsinki.

\section{Data Collection}

Physical Activity Measures. The combined heart rate and movement sensor attached to the participant's chest measured heart rate and uniaxial acceleration of the trunk in 15-second intervals (Brage, Brage, Franks, Ekelund, \& Wareham, 2005). The wrist accelerometer worn on the non-dominant wrist recorded triaxial acceleration at 60 Hertz. Participants were instructed to wear both waterproof monitors continuously for six full days and nights during free-living conditions, including during showering and while they were sleeping.

During the baseline clinic visit, participants performed a ramped treadmill test to establish their individual heart rate response to a submaximal exercise test (Brage et al., 2007). These measurements produced calibration parameters that were used in a branched equation model of PAEE (Brage et al., 2004). Heart rate data collected during free-living was pre-processed to eliminate potential noise (Stegle, Fallert, MacKay, \& Brage, 2008), following which the branched equation model was applied to calculate instantaneous PAEE $\left(\mathrm{J} \cdot \mathrm{min}^{-1} \cdot \mathrm{kg}^{-1}\right)$. This inference has been validated against 
intensity from indirect calorimetry (Strath, Brage, \& Ekelund, 2005; Thompson, Batterham, Bock, Robson, \& Stokes, 2006) and volume from doubly labeled water in several populations (Villars et al., 2012), including a sample of UK men and women in whom the technique was shown to explain $41 \%$ of the variance in free-living PAEE as well as no mean bias (Brage et al., 2015).

The wrist accelerometer data was processed using pampro, an open-source software package (White, 2018). The triaxial acceleration was auto-calibrated to local gravitational acceleration using a method described elsewhere (van Hees et al., 2014). Non-wear time was defined as time periods where the standard deviation of the acceleration in each of the three axes fell below 13mg for over an hour, inferring that the device was completely stationary (van Hees et al., 2011). When a non-wear period was detected, it was removed from the analyses. The magnitude of acceleration was calculated using Vector Magnitude (VM) (expressed in milli-g/mg) per sample:

$$
\mathrm{VM}(\mathrm{X}, \mathrm{Y}, \mathrm{Z})=\sqrt{\left(X^{2}+Y^{2}+Z^{2}\right)}
$$

VM, or Euclidean Norm, can be interpreted as the magnitude of acceleration the device was subjected to at each measurement, which includes gravitational acceleration. Any potential noise component in the high-frequency domain was filtered out by a 20 Hertz low-pass filter. To isolate the movement-related acceleration, we also applied a high-pass Butterworth filter to the VM signal at 0.2 Hertz (therefore treating gravity as a low-frequency component) naming the resulting metric Vector Magnitude High-Pass Filtered (VM HPF, expressed in $\mathrm{mg}$ ) (van Hees et al., 2011; White et al., 2016). VM HPF is commonly used as a proxy of acceleration resulting from human movement, has high validity (van Hees et al., 2013), and was the primary description of wrist movement in the following analyses.

To isolate the gravitational acceleration for each axis, we applied a low-pass filter $(0.2$ Hertz) to each of the three axes $(x, y$, and $z)$. The residual acceleration signal can be interpreted as a measurement of the rotated gravitational field vector which can then be used to determine the accelerometer's pitch and roll orientation angles. Pitch and roll of the device were derived according to these formulae:

$$
\begin{aligned}
\text { Pitch } & =\frac{-\tan ^{-1}\left(\frac{Y}{\sqrt{X^{2}+Z^{2}}}\right) * 180}{\pi} \\
\text { Roll }= & \frac{-\tan ^{-1}\left(\frac{X}{\sqrt{Y^{2}+Z^{2}}}\right) * 180}{\pi}
\end{aligned}
$$

As the monitor was mounted in such a way that the $x$-axis was aligned in anatomically opposite directions for left- and righthanded participants, we multiplied it by -1 for all left-handed participants who wore the monitor on their right wrists to align with the anatomical coordinate system defined above (examples of untransformed data shown in Supplementary Figure 2 [available online]). Consequently, positive pitch indicates upwards position of the arm (hand above elbow), while positive roll indicates the lateral (radial, thumb) side of the arm being higher than the medial (ulnar, pinky) side of the arm. Figure 1 illustrates these concepts.

All derived signals were summarized to a common time resolution of one observation per hour. This window length was chosen since we were mostly interested in observing changes at a diurnal level, rather than variations within the hour.

Using the combined-sensing measurements, participants were stratified by average activity energy expenditure into three equal tertiles: lower active $\left(\leq 39 \mathrm{~J} \cdot \mathrm{min}^{-1} \cdot \mathrm{kg}^{-1}\right)$, medium $\left(40-56 \mathrm{~J} \cdot \mathrm{min}^{-1} \cdot \mathrm{kg}^{-1}\right)$, and upper $\left(\geq 57 \mathrm{~J} \cdot \mathrm{min}^{-1} \cdot \mathrm{kg}^{-1}\right)$. These activity estimates were calculated for each participant for each day of the week and then averaged, allowing us to generate a activity-level stratification based on the individual weekly average.

Similarly, we calculated estimates of time spent in sedentary (i.e., sitting or reclining) by detecting bouts where pitch (i.e., forearm elevation) is $\geq 15^{\circ}$ below the horizontal, while acceleration is minimal (VM HPF $\leq 47.61 \mathrm{mg}$ ). This is based on principles from previously developed methodology which derives sedentary time estimates from wrist accelerometry data (i.e., sedentary sphere methodology [Rowlands et al., 2016]), as well as estimations of physical activity energy expenditure in free-living using wrist accelerometry (White et al., 2016). The latter defined the acceleration threshold (VM HPF $=$ $47.61 \mathrm{mg}$ ) equivalent to 1.5 gross METs $\left(\mathrm{PAEE}=35.5 \mathrm{~J} \cdot \mathrm{min}^{-1} \cdot \mathrm{kg}^{-1}\right.$ ) as the cut-off for sedentary behavior (7). Data in lower latitudes (i.e., $<-15^{\circ}$ from the horizontal) suggest hanging of the arm, associated to standing behaviors and are hence not classified as sedentary time. Equally, if the mean levels (VM HPF) over a minute fell into the light, moderate or vigorous category, they were not classified as sedentary behavior.

Using the diurnal profiles derived from the cohort, we studied differences based on sex, age, activity levels, BMI, and time of the day.

\section{Statistical Analyses}

We computed descriptive statistics (mean, median, standard deviation, minimum, maximum, and variance) for the participants in this analysis. We examined wear-time distributions using the Friedman test for time-of-day (00:00-05:59, 06:00-11:59, and so on in sixhour periods) and tested the differences in weekdays versus weekend days using Wilcoxon signed ranks. These tests were performed in men and women separately. Mean acceleration differences (VM $\mathrm{HPF}$ ) were examined using ANOVA for time of the day and day of the week. Differences between men and women are shown by using box plots, providing information about the median, inter-quartile range, minimum and maximum. We analyzed the differences between different BMI groups (underweight $\leq 18.5 \mathrm{~kg} / \mathrm{m}^{2}$, normal weight $18.5-24.9 \mathrm{~kg} / \mathrm{m}^{2}$, overweight $25-29.9 \mathrm{~kg} / \mathrm{m}^{2}$, obese 30 $34.9 \mathrm{~kg} / \mathrm{m}^{2}$, and severely obese $\geq 35 \mathrm{~kg} / \mathrm{m}^{2}$ ) in both sexes based on pitch, roll, VM HPF, and PAEE. Similarly, we conducted the analysis based on age group and PAEE levels. These summary statistics were computed at an hourly level after collapsing information derived on a 15 -second time window.

Furthermore, we tested for differences in time spent in sedentary time across the different BMI populations using 3-way ANOVA and adjusting for age and sex.

Statistical tests were performed using Python (3.6.2) and Stata (v14, StataCorp, College Station, TX).

\section{Results}

Among the 2,043 participants, a total of 286,020 person-hours were included in our analysis, or an average of 5.8 days per participant. As shown in Table 1, PAEE was higher in men although both groups had large standard deviations. However, wrist movement was similar between genders but mean BMI was larger in men than in women for this cohort.

Figure 2A shows pitch and roll distributions for men and women; a two-dimensional plot of pitch and roll is shown in Supplementary Figure 3 (available online). There is higher occurrence of pitch and roll positions around $0^{\circ}$ and the roll distribution is 
Table 1 Characteristics of Participants by Sex $(n=2,043)$

\begin{tabular}{lcc}
\hline & Men & Women \\
\hline $\mathrm{N}$ & 953 & 1090 \\
Age (years) & $50.9(7.3)$ & $50.5(7.1)$ \\
Height $(\mathrm{m})$ & $1.78(0.07)$ & $1.64(0.06)$ \\
Weight $(\mathrm{kg})$ & $86.2(14.1)$ & $70.8(14.3)$ \\
BMI $\left(\mathrm{kg} \cdot \mathrm{m}^{-2}\right)$ & $27.2(4.2)$ & $26.4(5.3)$ \\
PAEE $\left(\mathrm{J} \cdot \mathrm{min}^{-1} \cdot \mathrm{kg}^{-1}\right)$ & $53.1(21.9)$ & $47.7(19.1)$ \\
Wrist movement, VM HPF $(\mathrm{mg})$ & $31.7(44.9)$ & $31.1(40.8)$ \\
\hline
\end{tabular}

Note. Values are $M(S D)$.

distinctly bimodal with an additional peak around $35^{\circ}$. Less common are extreme anatomical forearm positions, e.g., arms up in the air reflected by a pitch $>60^{\circ}$, or the radial (thumb) side of the arm turned inwards and downwards as indicated by less roll data below $-45^{\circ}$.
Figures $2 \mathrm{~B}$ and $2 \mathrm{C}$ shows the differences among different age groups for average sedentary time and PAEE respectively. PAEE declines with age in both men and women, and there is a tendency for the wrist measure of sedentary time to increase with age, showing a close inverse relationship between these two measures.

\section{Relation Between Wrist Movement and Forearm Postures, and Physical Activity Energy Expenditure}

Figure 3 shows differences in wrist measures by tertile of physical activity energy expenditure; more active individuals spend more time in low-pitch (below horizontal) postures; less active participants tend to be spending more time in postures that suggest sedentary behaviors, such as sitting or reclining. Whilst roll angles differ by activity level in women, there is almost no difference between groups in men; differences in wrist movement, however, are very clear in both genders.

Pitch/Roll distribution by sex; Fenland Study ( $n=2043$ )

A

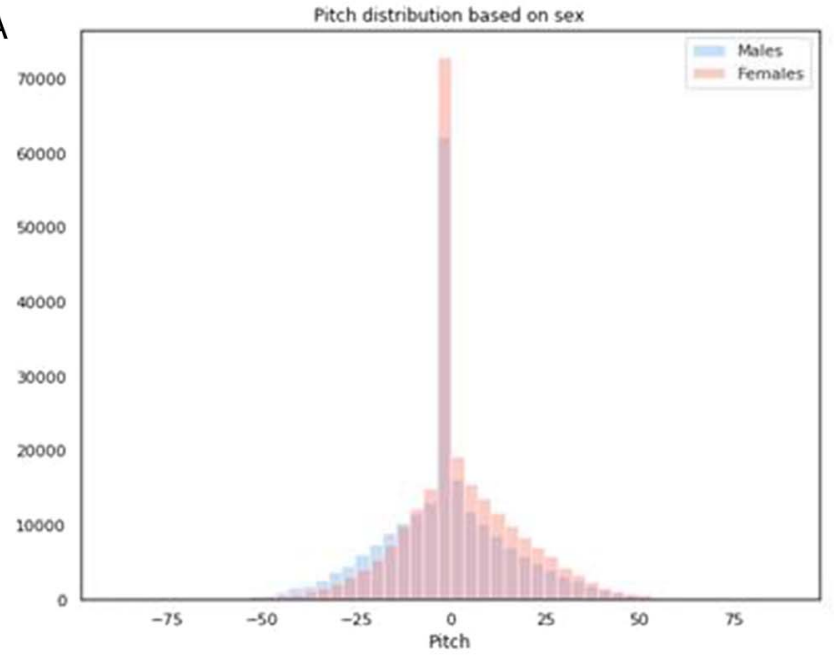

B Sedentary time by age and sex; Fenland Study $(n=2043)$

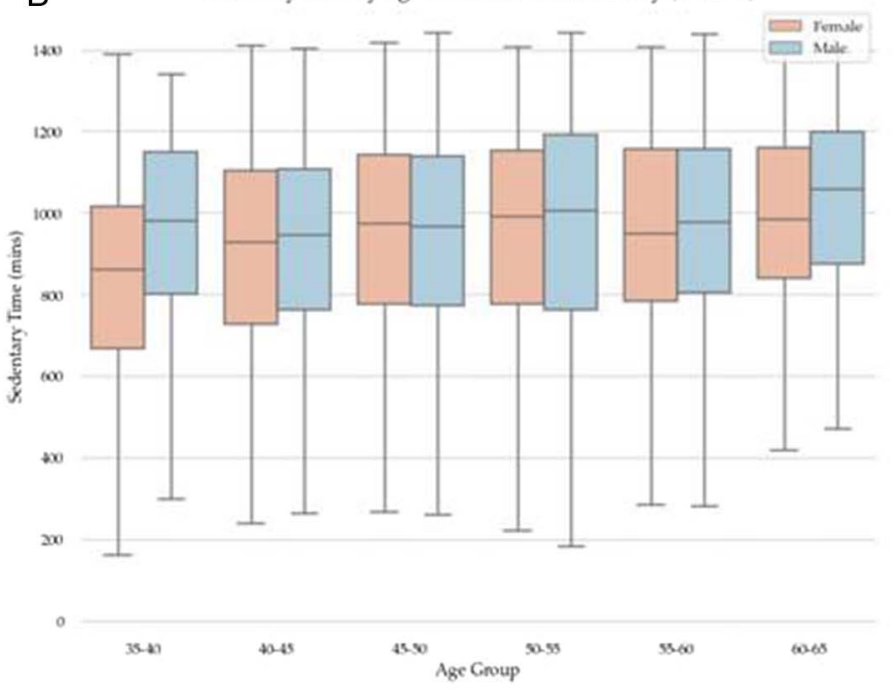

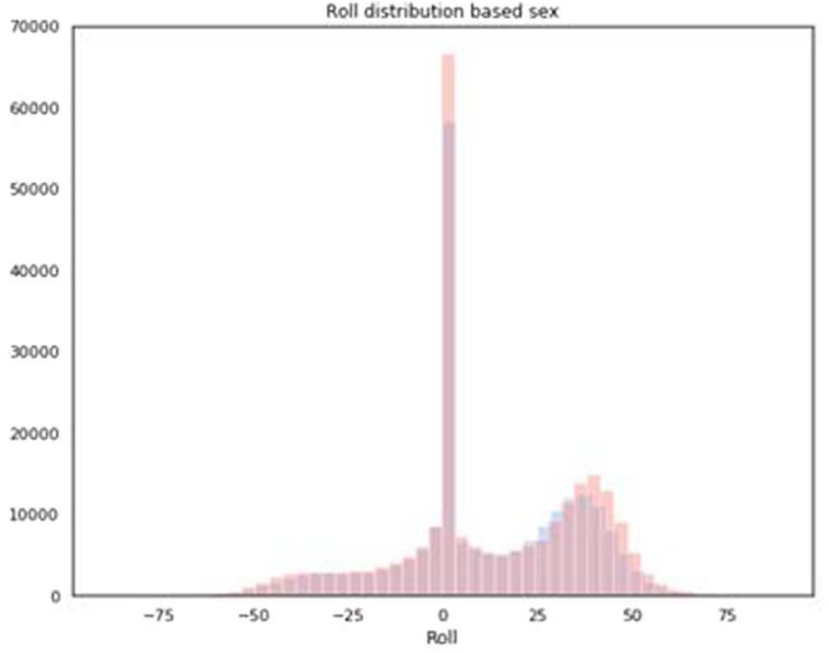

C

PAEE by age and sex; Fenland Study $(n=2043)$
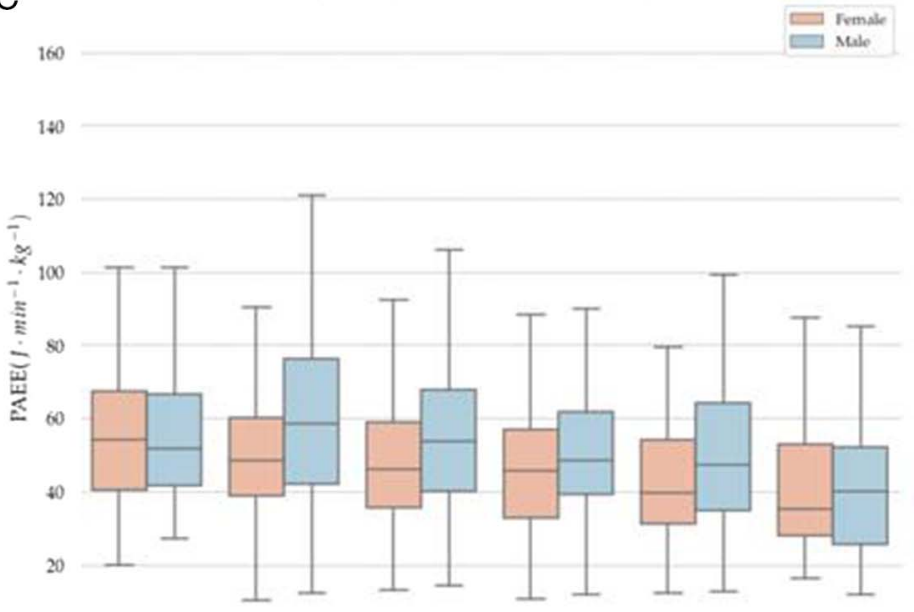

Figure 2 - Pitch and roll (A) distribution among participants, and box plots for time spent sedentary (B) and PAEE (C) by age group and sex $(n=2,043)$. 

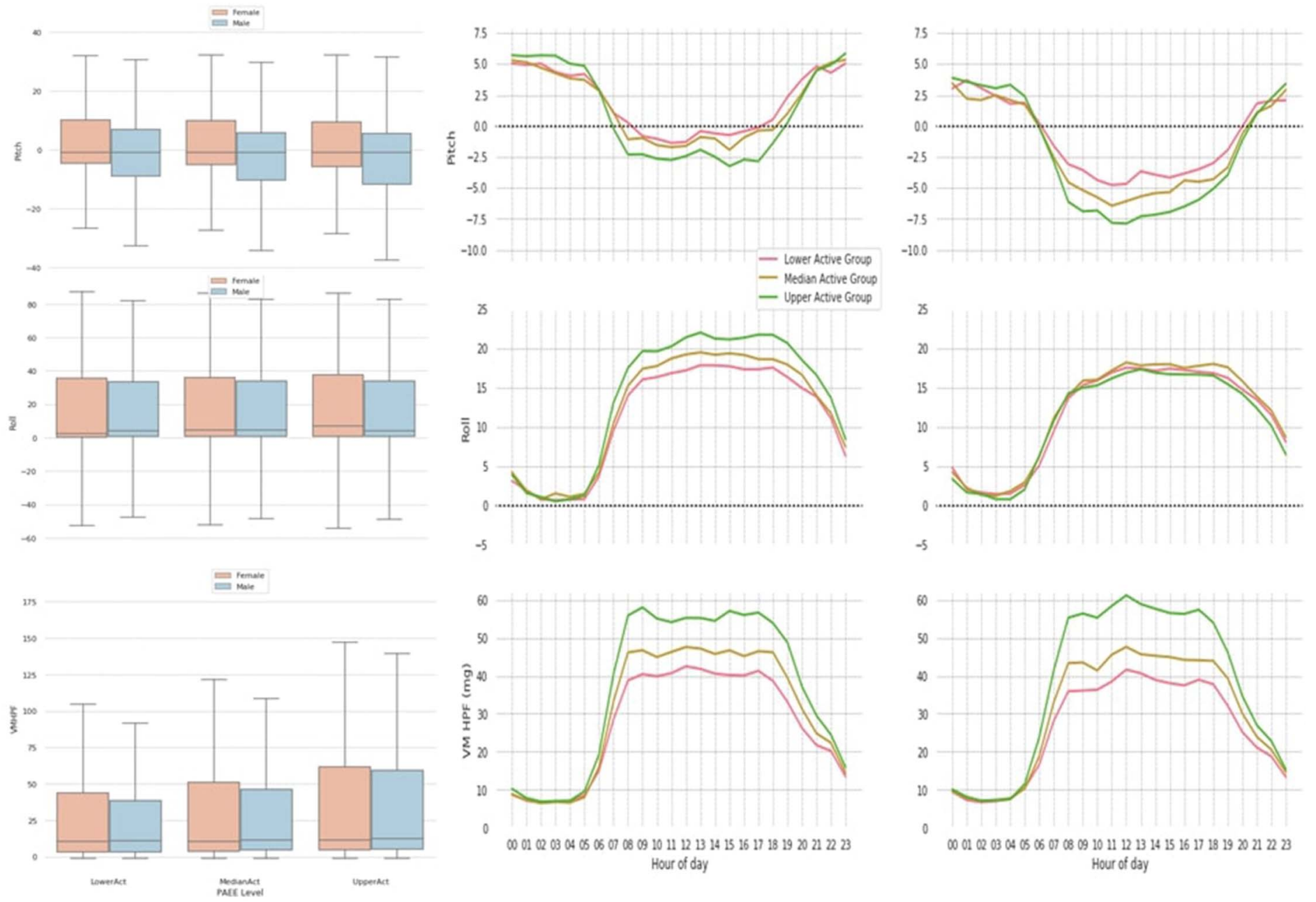

Figure 3 - Pitch (top panels), Roll (middle panels), and Vector Magnitude High-Pass Filtered (VM HPF) by physical activity energy expenditure level (lower, medium, or upper) and gender (A), and diurnal profiles (hourly averages) by time of day in women and men (B).

Some of the most visually striking results regarding the role of posture on physical activity behaviors can be seen in the threedimensional time-lapse plots that appear on the online supplementary online material of this paper (see Supplementary Video 1 [available online]). A schematic representation of these time-lapses is presented in Figure 4 at four times of the day.

\section{Diurnal Profile Differences by Sex and Age}

Figure 5 shows the distribution of pitch, roll, and movement intensity across the day, stratified by sex and age group. We observe differences between age groups within sex, but also differences between men and women within age groups. Most differences between men and women occur during the working hours (8 AM to $6 \mathrm{PM}$ ) of the day, with little differences at night although women generally keep their arms at slightly higher pitch throughout the 24 hours. Some of the biggest differences between age groups in both sexes happen during the early hours of the morning and late hours of the evening. Forearm angles differ more between age groups in men (lower pitch in older during working hours), and gender differences in pitch and roll profiles are most apparent among the 35- to 40-year-old age group.

\section{Pitch and Roll Profiles Differ on Weekends Versus Weekdays}

Figure 6 shows average pitch, roll, and movement intensity across the day, separately for each day of the week, and stratified by sex. The variation between weekdays at a population level is minimal, but they differ from the diurnal profiles at the weekend and particularly among sexes. A visible shift on weekend days towards later hours of the morning suggests a "later start" to the day, and later bed times on Friday and Saturday nights. The most extreme postural contrast are seen for pitch angles in men which reach the lowest level at the weekend (around $-10^{\circ}$ ) in parallel to highest level of movement; pitch in women is also lower in the weekend but only to the weekday level of the men (around $-5^{\circ}$ ) but with a similar level of movement as men.

\section{Wrist Accelerometry Profiles by Gender and BMI}

The differences in mean VM HPF between the different BMI groups are striking with obese individuals moving considerably less than normal-weight but equally notable are differences in pitch and roll profiles (Figure 7). Differences among groups were more 

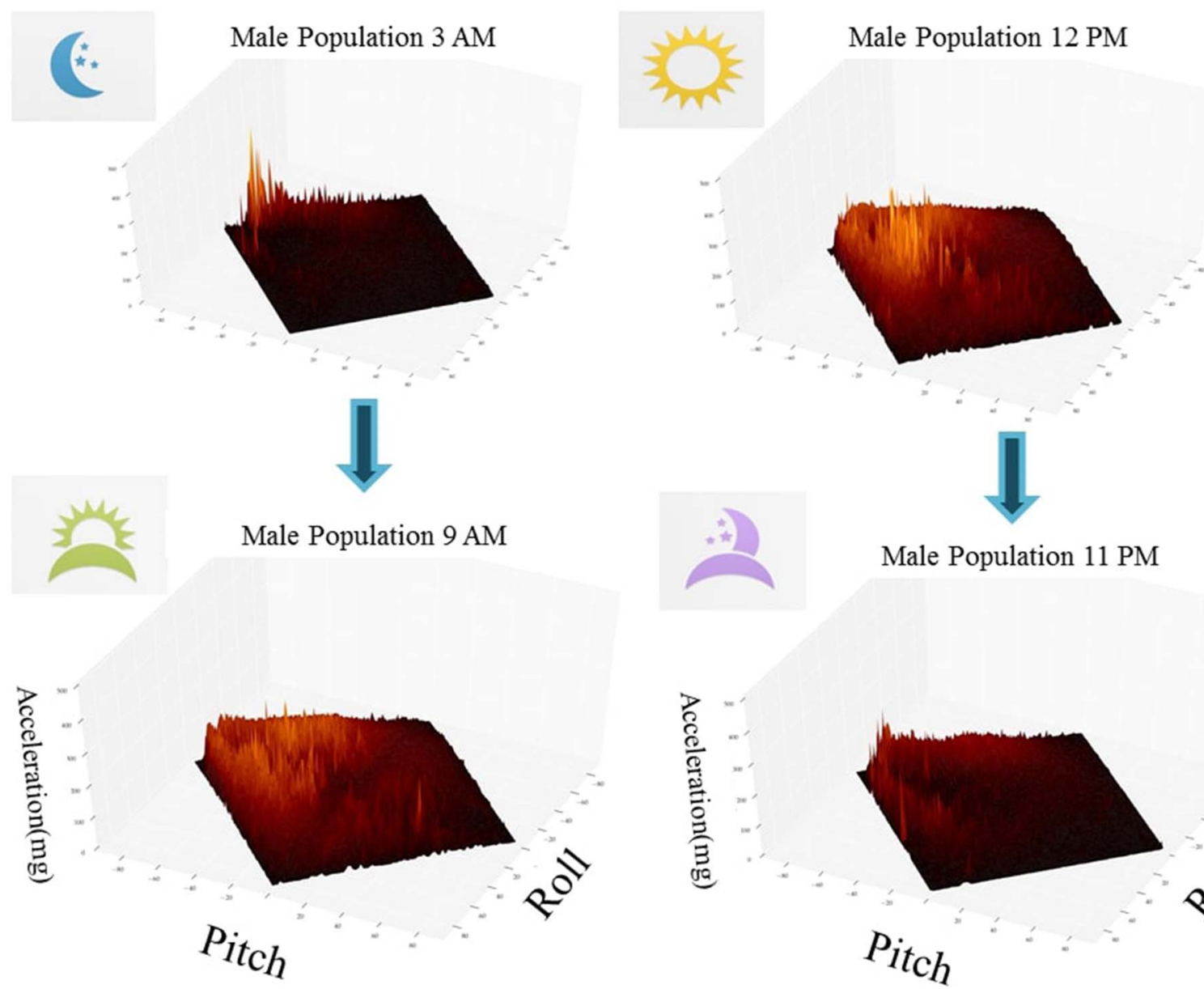

Male Population $11 \mathrm{PM}$

Figure 4 - Schematic representation of time-lapse diurnal change in Pitch and Roll angular profiles and their associated acceleration signal (Vector Magnitude High-Pass Filtered, in $\mathrm{m} g$ ). All plots have been normalized. (Figure derived from the male population of this analysis $n=953$ ). Full videos for both genders are available in online Supplemental Material.

apparent in men than in women when considering the diurnal profile. Somewhat surprisingly, given higher movement is generally occurring at the lower pitch angles (Figure 3), overweight and obese individuals spend more time with their arms in this space but they just do not seem to move as much. The underweight women's pitch and roll profile are very different to that observed in the severely obese men, suggesting that the higher level of mean physical activity in this group is also related to a very different set of activities. These observations are supported by stark differences on the average time spent in sedentary behaviors stratified by sex and BMI category, where non-obese participants spent considerably less time in sedentary behaviors than obese participants, particularly women. Also, the profiles observed in obese men closely resemble that observed in the oldest age group as presented in Figure 5. We confirmed differences across different BMI groups for average time spent in sedentary behaviors, following adjustment for age and sex. We found that moderately obese participants spent significantly more time in sedentary behaviors than normalweight participants $(p<.001)$, and so did severely obese $(p<.001)$ and even overweight participants $(p<.001)$. We also found a strong significant difference between overweight and moderately obese participants $(p=.0001)$; however, differences between normal-weight and underweight participants were not statistically significant $(p=.57)$.

\section{Discussion}

In this paper, we have explored the physical space in which physical activity occurs and described population differences in wrist movement and posture between men and women, age groups, BMI categories, and physical activity levels in a population sample of UK adults. Although higher activity was associated with lower pitch profiles, we observed the apparent paradox that older and more obese individuals who as groups are generally less active also spend more time at these postures, indicating that these groups either perform different types of activities or perform them at slower pace.

Vector magnitude of movement intensity and pitch-roll angular features can all be considered direct measures of human behavior, rather than estimates, as there is very little inference involved in deriving them; they have biomechanical meaning in their own right as also illustrated in Supplementary Figure 1 (available online). The estimate of sedentary time, on the other hand, is not a direct measure but an estimate resulting from an inference but we have included it here to demonstrate the utility of combining directly measured features. Including movement as well as pitch, roll (both indicating posture), and sedentary time estimates in our analysis allowed us to more comprehensively examine differences in human behavior between time-of-day and weekdays 
A
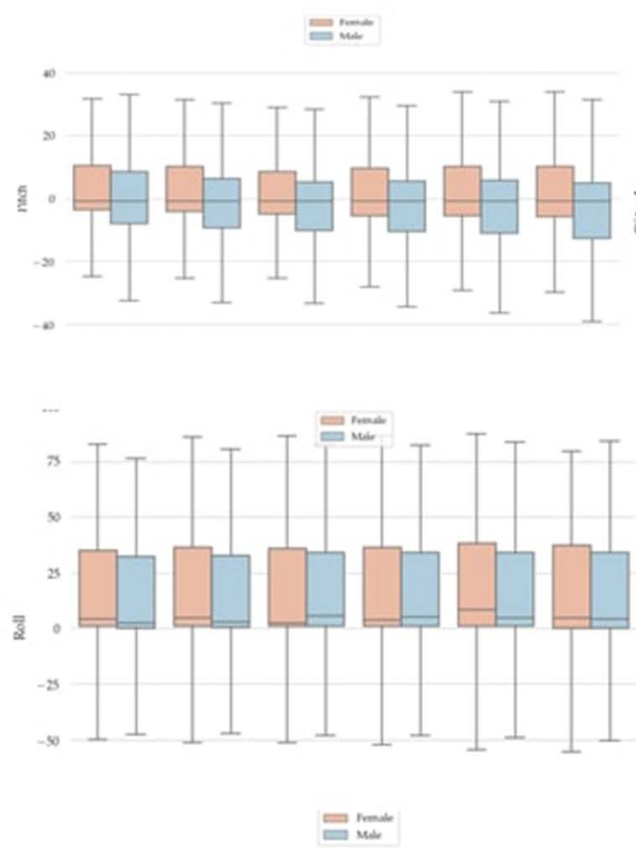

20

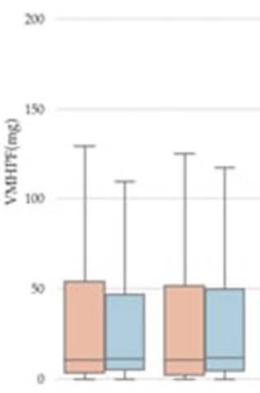

35-40

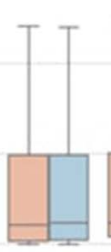

$450 \mathrm{AB}$

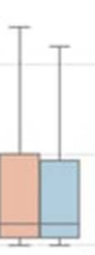

sass

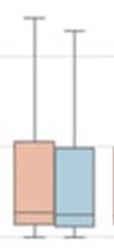

3500 now
B Female Mean Pitch, Roll, VM HPF based on time of day and age Male Mean Pitch, Roll, VM HPF based on time of day and age
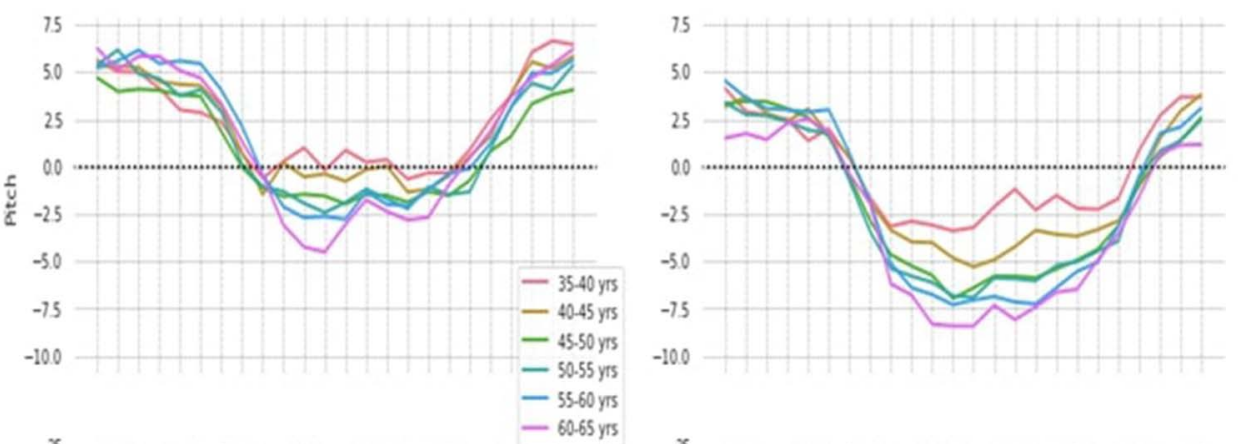

$-100$

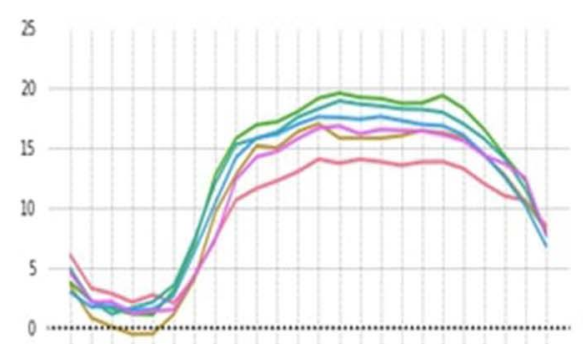

$-5$

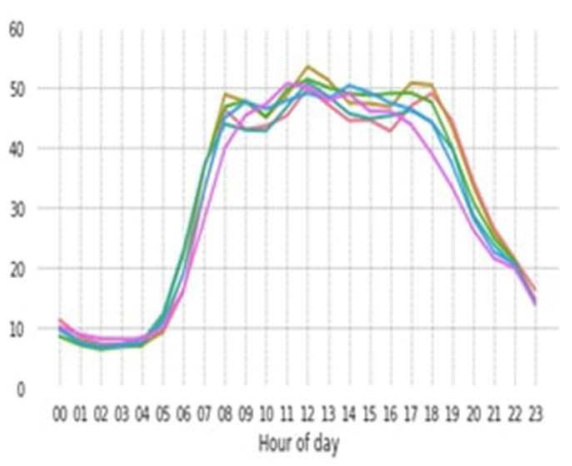

Figure 5 - Pitch (top panels), Roll (mid panels), and Vector Magnitude High-Pass Filtered (bottom panels) profiles (hourly averages) by time of the day and age group (from 35-40 to 60-65 years old) in women (middle column) and men (right column). Left column (A) shows participant-level summary data.

and weekends, and illustrates the importance of taking all these features into consideration for large-population studies. Nonsurprisingly, our results suggest different wake-up times between weekdays and weekends; participants seem to wake up later during the weekends than weekdays. This information is of interest particularly given recent research suggesting that sleep irregularity may be a risk factor for cardio-metabolic disease (Arora et al., 2013). The large differences in movement and postural measures between weekdays and weekends suggest differences in the type of activities that participants partake in between weekdays and weekends. These differences are particularly striking when comparing women and men. We found that women spend more time with their forearm elevated above horizontal than men do (53\% of their time vs. $36 \%$ for men). Similarly, the pitch and roll profiles coincide with increases in movement around noon of the weekend days, pointing towards a behavioral pattern that could be suggestive of "weekend warrior" lifestyle, where participants tend to do most of their physical activity during the weekend. Further inspection of the data through visualization techniques (Figure 4 and associated video files) suggests that the activities participants engaged in strongly depended on time-of-day; it is apparent that the relative occupation of different physical spaces and the relationship between postures and movement changes drastically depending on the time of the day, indicative of engagement in different activity types.

We observed differences between men and women across most other substrata for both movement (vector magnitude) and posture (pitch and roll) measures, suggesting that men spent more time in postures that may be suggestive of sedentary behavior than their female counterparts (sitting down, lying down). The inferred time estimate for sedentary behaviors (from vector magnitude and pitch), largely based on the methodology previously described by Rowlands et al. (2016), indicated that this was by far the most dominant behavior across the whole population ( $\sim 17$ hours/day). However, younger individuals tended to spend less time than their older counterparts in these sedentary behaviors (suggesting more active lifestyles), and even starker differences were observed between different BMI groups; individuals with higher BMI spent the most time in sedentary behaviors, and we statistically confirmed that this was independent of age and sex. 

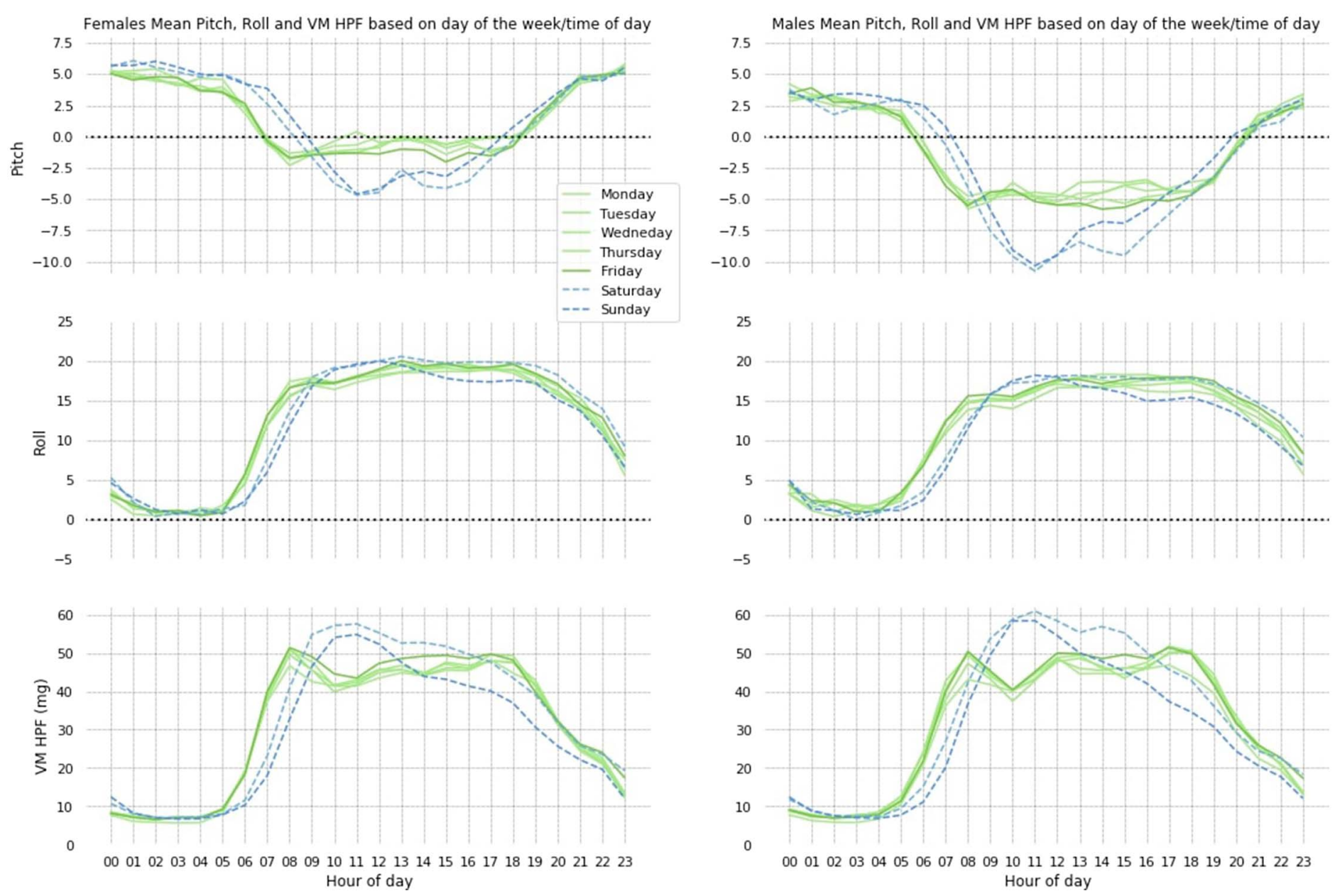

000102030405060708091011121314151617181920212223 Hour of day

Figure 6 - Differences in Pitch, Roll, and Vector Magnitude High-Pass Filtered (hourly averages) based on day of the week (solid lines indicate weekdays [green in online], dashed lines indicate weekends [blue in online]) and time of the day in women and men.

Movement and PAEE were both lower in the older age groups, a similar result to that observed in other population studies (Brage et al., 2019; Doherty et al., 2017; Troiano et al., 2008). We observed that older participants (60- to 65year-old age group) spend a large proportion of their time in postures that are similar to those with high BMIs, particularly in men. What was slightly paradoxical was that older and obese individuals spend more time at pitch angles generally associated with higher activity (i.e., with the arm below horizontal). As both movement and pitch are direct measurements of what the arm is physically doing, these results indicate true differences in activities, either as type or intensity or both. Using the sedentary time estimation methodology, it was suggested that older and heavier individuals spent more time in sedentary behaviors. Future inference work on raw non-dominant wrist acceleration signals may further elucidate other differences, for example in the specific type of activity performed, including the separation of awake sedentary behavior and sleep.

Strengths of our study includes its standardized placement and 24-hour wear protocol which ensured greater certainty in the orientation of the accelerometer on each participant; that said, it is possible that some participants may have removed and replaced their device during the monitoring period. Still our results may provide guidance on probable axis orientation to other studies such as UK Biobank which do not have strict device orientation protocols. Another strength was that both wrist acceleration and PAEE was assessed simultaneously, thus providing more accurate stratification by PAEE levels; however a limitation of our work is that we only measured physical activity during one week of monitoring, and this may not be representative of habitual behavior in this population. Another potential limitation is the separation between static and dynamic wrist acceleration; as has been previously addressed, the highand low-pass filter parameters does not perfectly discriminate between static and dynamic and a small proportion of real movement will be missed during rapid rotations (O'Donnell et al., 2017). Nonetheless, this is likely to only bias the movement differences we observe towards the null, since younger and slimmer individuals are more able to produce more rapid movements, and it will likely not impact much on the postural measures, as the gravitational acceleration component is several orders of magnitude larger than residual movement in the lowpass filtered signal, thus still returning a valid estimate of the relative distribution of gravity in the three axes. Finally, in this work we describe hourly averages of behavior and movement; however, there are alternatives to describing the underlying variability of these hourly-periods based on distributional statistics that were not explored in this paper. 
A Pitch, Roll, VMHPF and Sedentary Time by BMI
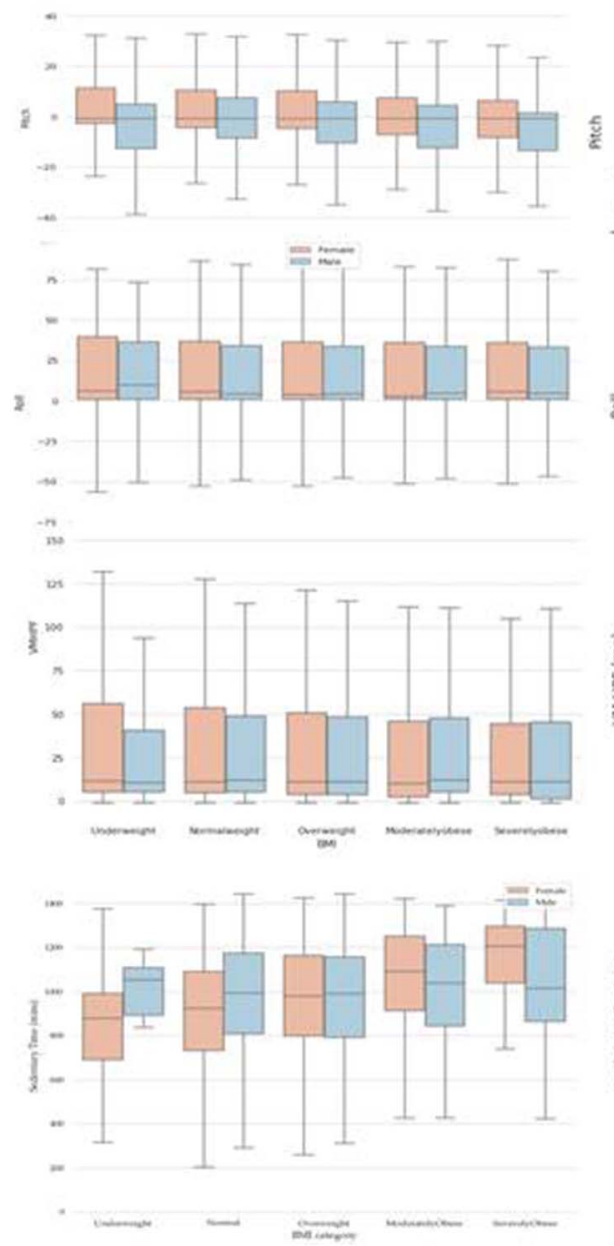

B Female Mean Pitch, Roll, VM HPF, Sedentary Time by BMI
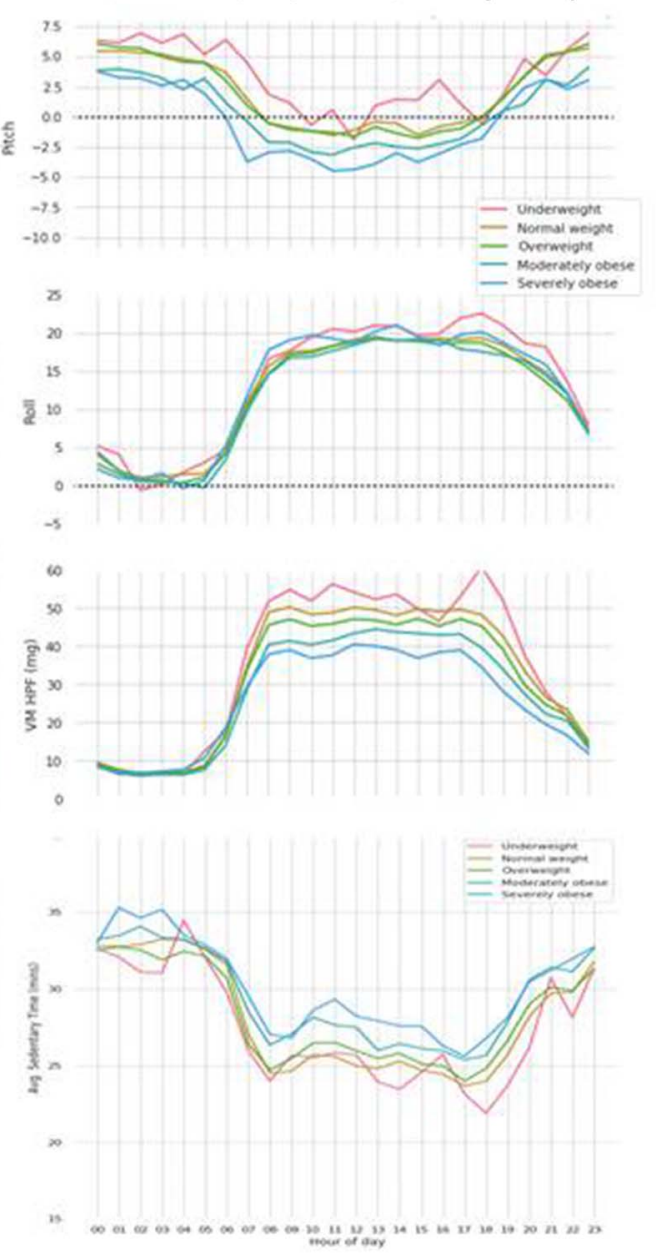

Male Mean Pitch, Roll, VM HPF, Sedentary Time by BMI
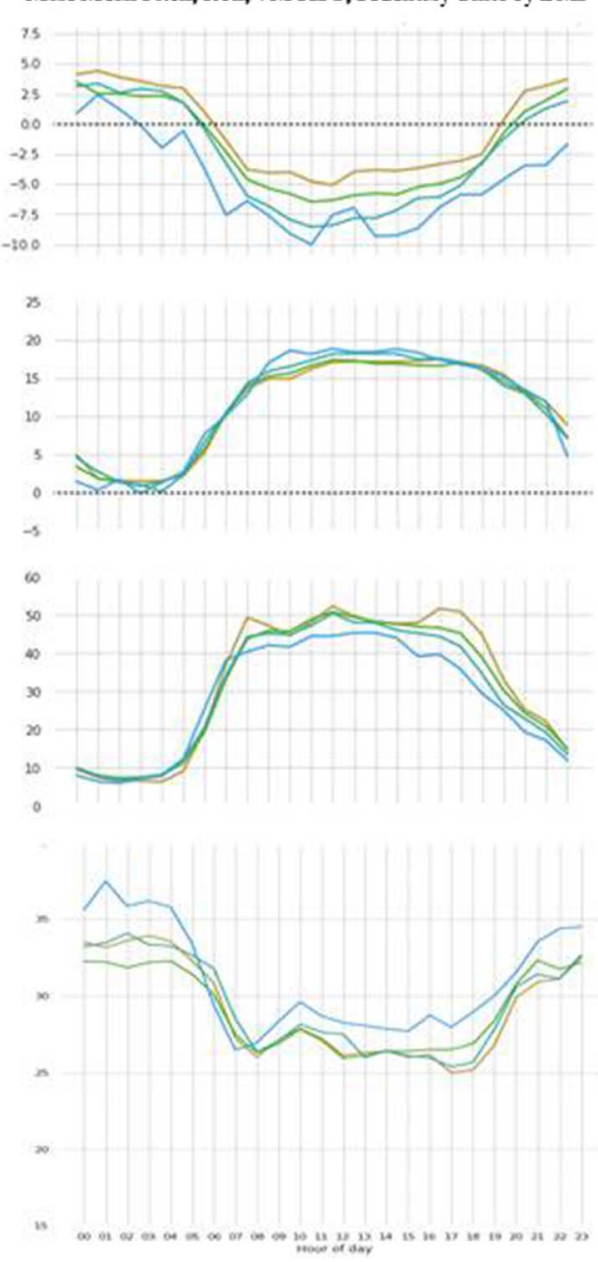

Figure 7 - Pitch (top panels), Roll (second row panels), Vector Magnitude High-Pass Filtered (third row panels), and sedentary time (bottom row panels) profiles (hourly averages) by time of the day in women and men, stratified by BMI categories (ranging from underweight [BMI: 16-18.5] to severely obese $[\mathrm{BMI} \geq 35])$.

\section{Conclusion}

In conclusion, we found that direct measures of accelerometryderived forearm angles provide biomechanically relevant information alongside the more well-established movement intensity metrics, such as vector magnitude, to better characterize objectively measured physical activity in free-living conditions. Movement is more likely to occur, on average, at forearm angles below horizontal; but despite older and heavier individuals moving less, these individuals still spend more time at lower forearm angles, suggesting population differences in style of movement which may be important for other health outcomes.

\section{Acknowledgments}

We would like to thank the participants who took part in this study. We also thank the principal investigators of the Fenland study for their work on this population and the functional teams of the MRC Epidemiology Unit at Cambridge (Field Epidemiology, Study Coordination, Data Management, and IT) for supporting this study. The authors would like to thank Lewis Griffiths, Stefanie Hollidge, and Antonia Smith for their assistance in the preparation of data for this study. The authors were supported by the UK
Medical Research Council (MC_UU_12015/1 and MC_UU_12015/3) and the NIHR Biomedical Research Centre in Cambridge (IS-BRC-121520014). We would also like to thank EPSRC and GlaxoSmithKline for their support through graduate fellowships (iCase 17100053).

\section{References}

Arora, T., Hosseini-Araghi, M., Bishop, J., Yao, G.L., Thomas, G.N., \& Taheri, S. (2013). The complexity of obesity in UK adolescents: relationships with quantity and type of technology, sleep duration and quality, academic performance and aspiration. Pediatric Obesity, 8(5), 358-366. PubMed ID: 23239604 doi:10.1111/j.2047-6310. 2012.00119.x

Brage, S., Brage, N., Franks, P.W., Ekelund, U., \& Wareham, N.J. (2005). Reliability and validity of the combined heart rate and movement sensor Actiheart. European Journal of Clinical Nutrition, 59(4), 561570. PubMed ID: 15714212 doi:10.1038/sj.ejcn.1602118

Brage, S., Brage, N., Franks, P.W., Ekelund, U., Wong, M.-Y., Andersen, L.B., .. Wareham, N.J. (2004). Branched equation modeling of simultaneous accelerometry and heart rate monitoring improves estimate of directly measured physical activity energy expenditure. 
Journal of Applied Physiology, 96(1), 343-351. PubMed ID: 12972441 doi:10.1152/japplphysiol.00703.2003

Brage, S., Ekelund, U., Brage, N., Hennings, M.A., Froberg, K., Franks, P.W., \& Wareham, N.J. (2007). Hierarchy of individual calibration levels for heart rate and accelerometry to measure physical activity. Journal of Applied Physiology, 103(2), 682-692. PubMed ID: 17463305 doi:10.1152/japplphysiol.00092.2006

Brage, S., Lindsay, T., Venables, M., Wijndaele, K., Westgate, K., Collins, D., ... Page, P. (2019). Descriptive epidemiology of energy expenditure in the UK: Findings from the National Diet and Nutrition Survey 2008 to 2015. BioRxiv, 542613. doi:10.1101/542613

Brage, S., Westgate, K., Franks, P.W., Stegle, O., Wright, A., Ekelund, U., \& Wareham, N.J. (2015). Estimation of Free-Living Energy Expenditure by Heart Rate and Movement Sensing: A Doubly-Labelled Water Study. PLoS ONE, 10(9), e0137206. PubMed ID: 26349056 doi:10.1371/journal.pone.0137206

Cho, M., \& Kim, J.-Y. (2017). Changes in physical fitness and body composition according to the physical activities of Korean adolescents. Journal of Exercise Rehabilitation, 13(5), 568-572. PubMed ID: 29114532 doi:10.12965/jer.1735132.566

da Silva, I.C., van Hees, V.T., Ramires, V.V, Knuth, A.G., Bielemann, R.M., Ekelund, U., ... Hallal, P.C. (2014). Physical activity levels in three Brazilian birth cohorts as assessed with raw triaxial wrist accelerometry. International Journal of Epidemiology, 43(6), 19591968. PubMed ID: 25361583 doi:10.1093/ije/dyu203

Doherty, A., Jackson, D., Hammerla, N., Plötz, T., Olivier, P., Granat, M.H., ... Wareham, N.J. (2017). Large scale population assessment of physical activity using wrist worn accelerometers: The UK Biobank Study. PLoS ONE, 12(2), e0169649. PubMed ID: 28146576 doi:10.1371/journal.pone.0169649

Dunstan, D.W., Howard, B., Healy, G.N., \& Owen, N. (2012). Too much sitting- A health hazard. Diabetes Research and Clinical Practice, 97(3), 368-376. PubMed ID: 22682948 doi:10.1016/j.diabres.2012. 05.020

Ekelund, U., Steene-Johannessen, J., Brown, W.J., Fagerland, M.W., Owen, N., Powell, K.E., . . . Lee, I.-M. (2016). Does physical activity attenuate, or even eliminate, the detrimental association of sitting time with mortality? A harmonised meta-analysis of data from more than 1 million men and women. The Lancet, 388(10051), 1302-1310. doi:10.1016/S0140-6736(16)30370-1

Hui-Wen Chuah, S., Rauschnabel, P.A., Krey, N., Nguyen, B., Ramayah, T., \& Lade, S. (2016). Wearable technologies: The role of usefulness and visibility in smartwatch adoption. Computers in Human Behavior, 65, 276-284. doi:10.1016/j.chb.2016.07.047

Lamkin, P. (2016). Wearable Tech Market To Be Worth \$34 Billion By 2020. Retrieved from https://www.forbes.com/sites/paullamkin/ 2016/02/17/wearable-tech-market-to-be-worth-34-billion-by-2020/ \#3a202b753cb5

Li, H., Wu, J., Gao, Y., \& Shi, Y. (2016). Examining individuals' adoption of healthcare wearable devices: An empirical study from privacy calculus perspective. International Journal of Medical Informatics, 88, 8-17. PubMed ID: 26878757 doi:10.1016/j.ijmedinf.2015. 12.010

Matthews, C.E., Chen, K.Y., Freedson, P.S., Buchowski, M.S., Beech, B.M., Pate, R.R., \& Troiano, R.P. (2008). Amount of time spent in sedentary behaviors in the United States, 2003-2004. American Journal of Epidemiology, 167(7), 875-881. PubMed ID: 18303006 doi:10.1093/aje/kwm390

Menai, M., van Hees, V.T., Elbaz, A., Kivimaki, M., Singh-Manoux, A., \& Sabia, S. (2017). Accelerometer assessed moderate-to-vigorous physical activity and successful ageing: results from the Whitehall II study. Scientific Reports, 7(1), 45772. doi:10.1038/srep45772
O’Connor, L., Brage, S., Griffin, S.J., Wareham, N.J., \& Forouhi, N.G. (2015). The cross-sectional association between snacking behaviour and measures of adiposity: the Fenland Study, UK. British Journal of Nutrition, 114(08), 1286-1293. doi:10.1017/S000711451500269X

O’Donnell, J., Hollowell, S., Salimi-Khorshidi, G., Velardo, C., Sexton, C., Rahimi, K., ... Doherty, A. (2017). Automated detection of sleep-boundary times using wrist-worn accelerometry. BioRxiv. doi:10.1101/225516

Patterson, R., McNamara, E., Tainio, M., de Sá, T.H., Smith, A.D., Sharp, S.J., ... Wijndaele, K. (2018). Sedentary behaviour and risk of allcause, cardiovascular and cancer mortality, and incident type 2 diabetes: a systematic review and dose response meta-analysis. European Journal of Epidemiology, 33(9), 811-829. PubMed ID: 29589226 doi:10.1007/s10654-018-0380-1

Rowlands, A.V., Yates, T., Olds, T., Davies, M., Khunti, K., \& Edwardson, C. (2016). Sedentary sphere: Wrist-worn accelerometerbrand independent posture classification. Medicine \& Science in Sports \& Exercise, 48(4), 748-754. PubMed ID: 26559451 doi:10. 1249/mss.0000000000000813

Steeves, J.A., Bowles, H.R., McClain, J.J., Dodd, K.W., Brychta, R.J., Wang, J., \& Chen, K.Y. (2015). Ability of thigh-worn ActiGraph and activPAL monitors to classify posture and motion. Medicine \& Science in Sports \& Exercise, 47(5), 952-9. PubMed ID: 25202847 doi:10.1249/MSS.0000000000000497

Stegle, O., Fallert, S.V., MacKay, D.J., \& Brage, S. (2008). Gaussian process robust regression for noisy heart rate data. IEEE Transactions on Biomedical Engineering, 55(9), 2143-2151. PubMed ID: 18713683 doi:10.1109/TBME.2008.923118

Strath, S.J., Brage, S., \& Ekelund, U. (2005). Integration of physiological and accelerometer data to improve physical activity assessment. Medicine \& Science in Sports \& Exercise, 37(Suppl. 11), S563-71.

Thompson, D., Batterham, A.M., Bock, S., Robson, C., \& Stokes, K. (2006). Assessment of Low-to-Moderate Intensity Physical Activity Thermogenesis in Young Adults Using Synchronized Heart Rate and Accelerometry with Branched-Equation Modeling. The Journal of Nutrition, 136(4), 1037-1042. PubMed ID: 16549471 doi:10.1093/jn/136.4.1037

Tremblay, M.S., Aubert, S., Barnes, J.D., Saunders, T.J., Carson, V., Latimer-Cheung, A.E., .. Chinapaw, M.J.M. (2017). Sedentary Behavior Research Network (SBRN)- Terminology Consensus Project process and outcome. International Journal of Behavioral Nutrition and Physical Activity, 14(1), 75. PubMed ID: 28599680 doi:10.1186/s12966-017-0525-8

Troiano, R.P., Berrigan, D., Dodd, K.W., Mâsse, L.C., Tilert, T., \& Mcdowell, M. (2008). Physical activity in the united states measured by accelerometer. Medicine \& Science in Sports \& Exercise, 40(1), 181-188. PubMed ID: 18091006 doi:10.1249/mss.0b013e31815a51b3

van der Ploeg, H.P., \& Hillsdon, M. (2017). Is sedentary behaviour just physical inactivity by another name? International Journal of Behavioral Nutrition and Physical Activity, 14(1), 142. PubMed ID: 29058587 doi:10.1186/s12966-017-0601-0

van Hees, V.T., Fang, Z., Langford, J., Assah, F., Mohammad, A., da Silva, I.C.M., . . B Brage, S. (2014). Autocalibration of accelerometer data for free-living physical activity assessment using local gravity and temperature: An evaluation on four continents. Journal of Applied Physiology, 117(7), 738-744. PubMed ID: 25103964 doi:10.1152/japplphysiol.00421.2014

van Hees, V.T., Gorzelniak, L., Dean León, E.C., Eder, M., Pias, M., Taherian, S., ... Brage, S. (2013). Separating movement and gravity components in an acceleration signal and implications for the assessment of human daily physical activity. PLOS ONE, 8(4), e61691. PubMed ID: 23626718 doi:10.1371/journal.pone. 0061691 
van Hees, V.T., Renström, F., Wright, A., Gradmark, A., Catt, M., Chen, K.Y., ... Franks, P.W. (2011). Estimation of daily energy expenditure in pregnant and non-pregnant women using a wrist-worn tri-axial accelerometer. PLoS ONE, 6(7), e22922. PubMed ID: 21829556 doi:10.1371/journal.pone.0022922

Villars, C., Bergouignan, A., Dugas, J., Antoun, E., Schoeller, D.A., Roth, H., ... Simon, C. (2012). Validity of combining heart rate and uniaxial acceleration to measure free-living physical activity energy expenditure in young men. Journal of Applied Physiology, 113(11), 1763-1771. PubMed ID: 23019315 doi:10.1152/ japplphysiol.01413.2011
White, T. (2018). Pampro: physical activity monitor processing. Retrieved from https://github.com/Thomite/pampro

White, T., Westgate, K., Hollidge, S., Venables, M., Olivier, P., Wareham, N., \& Brage, S. (2019). Estimating energy expenditure from wrist and thigh accelerometry in free-living adults: a doubly labelled water study. International Journal of Obesity, 43, 2333-2342. doi:10.1038/ s41366-019-0352-x

White, T., Westgate, K., Wareham, N.J., \& Brage, S. (2016). Estimation of physical activity energy expenditure during free-living from wrist accelerometry in UK adults. PLoS ONE, 11(12), e0167472. PubMed ID: 27936024 doi:10.1371/journal.pone.0167472 